\title{
HIPERTENSÃO ARTERIAL, ATIVIDADE FÍSICA E EXCESSO PONDERAL EM ADOLESCENTES DO NORDESTE DO BRASIL
}

\section{Arterial hypertension, physical activity excess ponderal in adolescents of northeast brazil}

\author{
Claudia dos Reis Lisboa ${ }^{1}$; Helena Alves de Carvalho Sampaio ${ }^{2}$; Francisco Jose Maia Pinto ${ }^{3}$ \\ ${ }^{1}$ Universidade do Estado do Rio Grande do Norte Departamento de Educação Física Rua Seu Né Bezerra, 1620 - casa 110 \\ Bairro Costa e Silva - Mossoró - RN - (84) 9904-8007 e (85) 8633-0102 - kallsport@yahoo.com.br \\ ${ }^{2}$ Universidade Estadual do Ceará - Curso de Mestrado Acadêmico em Saúde Publica - Av. Dede Brasil, 1700 - Bairro Serrinha \\ Fortaleza - CE - (85) 3101-9600 e (85) 8802-8796 - dr.hard@terra.com.br \\ ${ }^{3}$ Universidade Estadual do Ceara - Curso de Mestrado Acadêmico em Saúde Publica - Av. Dede Brasil, 1700 - Bairro Serrinha \\ Fortaleza - CE - (85) 3101-9600 e (85) 8817-1908 - maiapinto@yahoo.com.br
}

Resumo: Ao se pensar na adoção de um estilo de vida saudável se desperta para a importância de conhecer a realidade de crianças e adolescentes em relação aos hábitos cotidianos e às prevalências de excesso ponderal e hipertensão arterial. O objetivo desse trabalho foi investigar a presença de hipertensão arterial entre adolescentes e sua associação com excesso ponderal e atividade física. Trata-se de um estudo transversal de base escolar que avaliou 565 adolescentes de 14 a 19 anos, ambos os sexos, matriculados na rede pública de ensino de Caucaia, região metropolitana de Fortaleza-CE. A pressão arterial foi aferida e classificada conforme as V Diretrizes Brasileiras de Hipertensão. Utilizou-se o Índice de Massa Corporal - IMC categorizado conforme tabela com percentis para idade e sexo segundo o National Center for Health Statistics (NCHS) e o Centers for Disease Control and Prevention (CDC). Para avaliar a atividade fisica dos participantes utilizou-se o International Physical Activity Questionnaire - IPAQ. Dos 565 adolescentes estudados, detectou-se alteração na pressão arterial em $45,3 \%$ sendo esse valor mais expressivo entre as meninas ( $53,3 \%$ ). Observou-se também a prevalência de $13,5 \%$ de excesso ponderal entre o grupo e a presença de atividade física adequada em $70,3 \%$ dos alunos. Conclui-se então que os níveis pressóricos dos adolescentes avaliados não foram associados à atividade física e ao estado nutricional, mas a alta prevalência de valores elevados demanda a investigação de outros fatores que possam estar associados a esta situação, a fim de direcionar ações de intervenção em curto prazo.

Palavras-chave: Hipertensão arterial, excesso ponderal, atividade física

Abstract: When we think about the adoption of a healthy life style we have to think about the importance to know the reality of children and adolescents in relation to the daily habits and the prevalence of weight excess and arterial hypertension. The aim of this study was to investigate the presence of arterial hypertension among adolescents and its association with weight excess and physical activity. It is a transversal cross-sectional study of the one school base which evaluated 565 adolescents,. They were 14-19 years old, both sexes, from the education public net scholl of Caucaia city, metropolitan area from of Fortaleza - CE,. The blood pressure was measured and classified according to Brazilian Guidelines of Hypertension. The weight excess body mass was evaluated using Body Mass Index (BMI), with categorization according to National Center for Health Statistics (NCHS) and the Centers for Disease Control and Prevention (CDC), which determine percentiles for age and sex. The International Physical Activity Questionnaire - IPAQ was used to evaluate the physical activity. Alterations in the blood pressure were detected in $45 ., 3 \%$ of the 565 studied adolescents, mainly among girls $(53.3 \%)$. The prevalence of weight excess was $13.5 \%$ and the presence of adequate physical activity was observed in $70.3 \%$ of the students. It was concluded that the blood pressure wasn't associated with physical activity and weight excess among evaluated adolescents, but the situation detected demands the investigation of another factors that can be associated, in order to direct actions of intervention asso soon is possible.

Keywords: Arterial hypertension, weight excess, physical activity

Aceito em 17/01/2011 - Revista de Educação Físíca 2011 Abr; 151:17-24. Rio de Janeiro - Brasil

\section{INTRODUÇÃO}

Atualmente, as discussões em Saúde Pública são focalizadas na busca do envelhecimento bem sucedido ressaltando-se a importância da adoção de um estilo de vida saudável desde a infância, prevenindo, retardando ou mesmo controlando o surgimento das doenças crônicas não transmissiveis (DCNT) uma vez desencadeadas ${ }^{(1)}$.

Ao se pensar na adoção de um estilo de vida saudável se desperta para a importância de conhecer a realidade de crianças e adolescentes em relação ao excesso ponderal e à hipertensão arterial. Essas prevalências muitas vezes se apresentam atreladas a um estilo de vida inadequado, caracterizado tanto por uma ingestão dietética inadequada como também pela baixa prática de atividade física ou sedentarismo ${ }^{(2-9)}$.

Entre os jovens o aumento da obesidade quase dobrou nos últimos 15 anos. Nos EUA $20 \%$ das 
crianças e $12 \%$ dos adolescentes 5 encontra-se hoje com peso excessivo o que aumenta o risco de enfermidades cardiovasculares quando adultos ${ }^{(10)}$

No Brasil a prevalência de excesso ponderal em crianças e adolescentes é de 17,0\% em meninos e 15,4\% em meninas e da obesidade 1,8\% e $2,9 \%$ respectivamente 11 . A prevalência de hipertensão arterial é de $8,2 \%$ para meninos e meninas apresentando frequente associação com sobrepeso e obesidade ${ }^{(10)}$.

Há casos de hipertensão arterial sem causas identificáveis principalmente em adultos ${ }^{(12)}$, porém há um aumento da prevalência de hipertensão arterial juvenil associada à obesidade por existir uma forte ligação entre elas e o metabolismo da insulina, tônus simpático, vagal, sistema circulatório e distúrbios do sono(10). A hiperinsulinemia, hiperleptinemia, distribuição centrípeta da gordura corporal e histórico familiar também são fatores de risco para hipertensão arterial na infância e adolescência(10), por isso a necessidade de se reduzir atividades sedentárias e incluir atividades físicas diárias e dieta adequada(13)

Em face da inter-relação apontada entre hipertensão arterial, excesso ponderal e falta de atividade física, e considerando a escassez de estudos direcionados para abordagem da temática em grupos populacionais constituídos por adolescentes, o objetivo do presente estudo foi investigar a presença de hipertensão arterial e sua associação com estado nutricional e atividade física entre adolescentes escolares.

\section{METODOLOGIA}

O estudo foi realizado em adolescentes matriculados em uma escola pública da cidade de Caucaia, integrante da região metropolitana de Fortaleza, capital do Ceará. Ocupando uma área de $1.228 \mathrm{~km}^{2}$, com uma população de 316.906 habitantes é o município mais populoso do estado depois de Fortaleza(11).

Para o presente estudo foi selecionada uma das 13 escolas de ensino médio pertencentes à rede pública estadual. Optou-se por escolher a escola com o maior número de alunos matriculados, que foi o Colégio Branca Carneiro de Mendonça.

Os critérios adotados para inclusão no estudo foram: ter idade entre 14-19 anos, independente do sexo, estar matriculado no ensino médio e não ter doença que inviabilizasse a prática de atividade física.

Dos 1783 estudantes que frequentavam a escola na época da coleta de dados (setembro a novembro de 2009) foram excluidos: 214 por se encontrarem fora da faixa etária estipulada para a pesquisa; 21 por possuírem doença limitante da prática de atividade física; 14 por não concordarem com a coleta dos dados antropométricos; 170 que resolveram abandonar o estudo uma vez iniciado e 799 que não concordaram em participar, não foram autorizados pelos pais ou não entregaram o termo de consentimento livre e esclarecido, assim, o estudo incluiu 565 alunos.

Antes de iniciar o mesmo foi submetido ao Comitê de Ética em Pesquisa com Seres Humanos da Universidade Estadual do Ceará e todos os participantes e seus responsáveis legais assinaram um termo de consentimento livre e esclarecido.

A coleta de dados foi realizada em três momentos. A pressão arterial foi aferida conforme recomendações do The Fourth Report on the Diagnosis, Evaluation, and Treatment of High Blood Pressure in Children and Adolescents 14 e quando omisso, de acordo com as $V$ Diretrizes Brasileiras de Hipertensão Arterial12. Foi utilizado um aparelho de pressão arterial automático com leitura digital, marca Geraterm, já validado e aprovado para uso clínico pela Sociedade Brasileira de Cardiologia conforme informações do fabricante. De acordo com o protocolo, a primeira aferição foi realizada em ambos os membros superiores e, para as aferições subseqüentes foi utilizado o membro que acusou maior valor nesta primeira aferição. A aferição ocorreu três vezes em cada momento, com intervalo mínimo de um minuto entre as medidas, sendo considerada a pressão arterial do indivíduo para aquele dia a média das duas últimas aferições. A pressão arterial considerada como sendo a do indivíduo foi a referente à média dos dias 1,2 e 3.

Para a classificação dos níveis pressóricos 
foi utilizado o valor médio das medidas aferidas, como descrito, sendo então tal média classificada de acordo com a tabela para valores correspondentes aos diferentes percentis de PA, que considera níveis desejáveis segundo sexo, idade e percentil de altura/idade. Os pontos de corte tanto para pressão arterial sistólica (PAS), como para pressão arterial diastólica (PAD), para essa população, estabelecidos em percentis, são: $\leq 90$ normal; entre 90 e 95 pré-hipertensão (ou sempre que a PA for maior que $120 / 80 \mathrm{mmHg}$, independente do percentil); entre 95 e 99 acrescido de $5 \mathrm{mmHg}$ hipertensão estágio 1; acima de 99 acrescido de $5 \mathrm{mmHg}$ hipertensão estágio $2^{(10)}$. Quando os percentis encontrados diferem, considerando PAS e PAD, a classificação é efetuada segundo o valor mais alto.

A avaliação do estado nutricional foi realizada através do índice de massa corporal (IMC). Foram obtidas medidas de altura (expressa em $\mathrm{cm}$ ) através do antropômetro marca Alturexata $\AA$, que possui capacidade de $2 \mathrm{~m}$ e intervalo de $0,5 \mathrm{~cm}$ e peso corporal (expresso em $\mathrm{kg}$ ) obtido através de uma balança eletrônica da Marca Camry Modelo ED307 com capacidade de $120 \mathrm{~kg}$ e precisão de até $0,5 \mathrm{~kg}$. Ambas as medidas foram obtidas segundo preconizações da OMS5. O IMC (expresso em kg/ $\mathrm{m}^{2}$ ) foi determinado a partir das duas medidas $e$ categorizado segundo o National Center for Health Statistics (NCHS), em colaboração com o Centers for Disease Control and Prevention (CDC), considerando idade e sexo: desnutrição quando a medida estiver abaixo do percentil 5 , eutrofia quando a medida estiver no intervalo de percentis $\geq 5$ e $<85$, sobrepeso quando a medida estiver no intervalo de percentis $\geq 85$ e $<95$ e obesidade quando a medida estiver igual ou maior que o percentil $95^{(15)}$.

Para avaliar a atividade física dos participantes, eles responderam ao International Physical Activity Questionnaire (IPAQ)(16). O IPAQ é um instrumento, recordatório semanal, desenvolvido com a finalidade de estimar o nível da prática da atividade física populacional, recomendado pela OMS em 25 paises, inclusive no Brasil desde 1998. É apresentado em duas versões, uma longa e outra curta, sendo essa última mais indicada para a população de crianças e adolescentes ${ }^{(17)}$.

Para a análise dos resultados o IPAQ classifica o indivíduo como muito ativo, ativo, irregularmente ativo ou então sedentário ${ }^{(17)}$. Segundo seus critérios um indivíduo muito ativo é aquele que pratica exercícios vigorosos 5 ou mais dias por semana, com pelo menos 30 minutos de duração cada sessão, ou 3 dias por semana de atividades vigorosas com pelo menos 20 minutos de duração cada sessão, adicionado de atividades moderadas 5 ou mais dias por semana, com pelo menos 30 minutos de duração cada sessão. $O$ indivíduo ativo é aquele que pratica atividades vigorosas 3 ou mais dias por semana, com o mínimo de 20 minutos de duração cada sessão ou então aquele que pratica atividades moderadas ou leves 5 dias por semana com pelo menós 30 minutos de duração cada sessão. $O$ indivíduo irregularmente ativo é aquele que realiza atividade física, porém insuficiente para ser classificado como ativo ou muito ativo. Os sedentários são caracterizados pela ausência de atividades físicas de qualquer tipo por pelo menos 10 minutos consecutivos.

As inter-relações entre hipertensão arterial foram efetuadas considerando o confronto da presença (pré-hipertensão, hipertensão estágio 1 ou hipertensão estágio 2) ou ausência (pressão arterial normal) de níveis pressóricos alterados com os indicadores de atividade física considerados ativos (muito ativo e ativo) e não ativos (irregularmente ativo e sedentário) e de estado nutricional classificados em sem excesso ponderal (desnutrição e eutrofia) e com excesso ponderal (sobrepeso e obesidade).

Os dados foram inseridos no programa estatístico Predictive Analytics Software for Windows - PASW18, versão 17.0. A análise estatística foi realizada através do teste Qui- quadrado $(x 2)$ ou exato de Fisher, adotando-se $p<0,05$, como nivel de significância.

\section{RESULTADOS}

Dentre os 565 alunos estudados, 214 (37,9\%) eram do sexo masculino e $351(62,1 \%)$ do sexo feminino.

A Tabela 1 mostra a distribuição dos alunos 
segundo categorização dos niveis pressóricos e sexo. Há uma alta prevalência de níveis pressóricos alterados (pré-hipertensão e hipertensão), principalmente no sexo feminino $(p=0,000)$.

Tabela 1. Distribuição dos alunos avaliados, segundo categorização dos niveis da pressão arterial e sexo.

\begin{tabular}{ccccccc}
\hline \multirow{2}{*}{ Pressão arterial* } & \multicolumn{2}{c}{ Masculino } & \multicolumn{2}{c}{ Feminino } & \multicolumn{2}{c}{ Total } \\
& N & $\%$ & N & $\%$ & N & $\%$ \\
\hline Normal & 134 & 62,6 & 164 & 46,7 & 298 & 52,7 \\
Pré hipertensão & 33 & 15,4 & 80 & 22,8 & 113 & 20,0 \\
Estágio 1 & 29 & 13,6 & 40 & 11,4 & 69 & 12,2 \\
Estágio 2 & 18 & 8,4 & 67 & 19,1 & 85 & 15.1 \\
\hline Total & 214 & 100,0 & 351 & 100,0 & 565 & 100,0 \\
\hline
\end{tabular}

*Segundo V Diretrizes Brasileiras de Hipertensão Arterial, 200612

A Tabela 2 exibe os achados referentes ao estado nutricional do grupo. A situação é similar entre os sexos $(p=0,803)$.

Tabela 2. Distribuição dos alunos avaliados, segundo estado nutricional e sexo.

\begin{tabular}{crrrrrr}
\hline \multirow{2}{*}{ Estado nutricional } & \multicolumn{2}{c}{ Masculino } & \multicolumn{2}{c}{ Feminino } & \multicolumn{2}{c}{ Total } \\
& N & \multicolumn{1}{c}{$\%$} & \multicolumn{1}{c}{ N } & \multicolumn{1}{c}{$\%$} & N & $\%$ \\
\hline Desnutrido & 20 & 9,4 & 26 & 7,4 & 46 & 8,1 \\
Normal & 164 & 76,6 & 280 & 79,8 & 444 & 78,6 \\
Sobrepeso & 17 & 7,9 & 37 & 10,5 & 54 & 9,6 \\
Obeso & 13 & 6.1 & 8 & 2,3 & 21 & 3,7 \\
\hline Total & 214 & 100,0 & 351 & 100,0 & 565 & 100,0 \\
\hline
\end{tabular}

*Determinado pelo índice de massa corporal - IMC e categorizado segundo $C^{15} C^{15}$

A Tabela 3 mostra a distribuição dos alunos segundo categorização de atividade física e sexo. A maioria de ambos os sexos apresenta um nível satisfatório de atividade (ativo ou muito ativo), sem diferença entre os sexos $(p=0,185)$.

Tabela 3. Distribuição dos alunos avaliados, segundo nível de atividade física e sexo.

\begin{tabular}{ccccrrr}
\hline \multirow{2}{*}{ Atividade fisica* } & \multicolumn{2}{c}{ Masculino } & \multicolumn{2}{c}{ Feminino } & \multicolumn{2}{c}{ Total } \\
& N & \% & N & $\%$ & N & $\%$ \\
\hline Sedentário & 26 & 12,1 & 30 & 8,5 & 56 & 9,9 \\
Insuficiente & 32 & 15,0 & 80 & 22,8 & 112 & 19,8 \\
Ativo & 128 & 59,9 & 220 & 62,7 & 348 & 61,6 \\
Muito ativo & 28 & 13,0 & 21 & 6,0 & 49 & 8,7 \\
\hline Total & 214 & 100,0 & 351 & 100,0 & 565 & 100,0 \\
\hline
\end{tabular}

*Guedes et. al. ${ }^{17}$
Os níveis pressóricos foram confrontados com a prática regular ou não de atividade física e com a presença ou não de excesso ponderal, como exposto, respectivamente, nas Tabelas 4 e 5 . Constatou-se não haver associação entre atividade fisica e pressão arterial, considerando o grupo todo (Qui-quadrado $=1,152 ; p=0,562$ ), sexo feminino: (Qui-quadrado $=0,161 ; p=0,923$ ) e sexo masculino (Qui-quadrado $=2,563 ; p=0,278$ ). Também não houve associação entre excesso ponderal e pressão arterial no grupo avaliado (Qui-quadrado $=1,217 ; p=0,270)$, mesmo considerando sexo feminino (Qui-quadrado $=0,906 ; p=0,341$ ) ou masculino (Fisher $=0,800 ; p=0,803$ ).

Tabela 4. Distribuição dos alunos estudados segundo categorias de atividade física, sexo e classificação de pressão arterial.

\begin{tabular}{cccccccr}
\hline & \multicolumn{4}{c}{ Pressão arterial } & \multicolumn{2}{c}{ Total } \\
Excesso ponderal & \multicolumn{2}{c}{ Alterada } & \multicolumn{2}{c}{ Não alterada } & \multicolumn{2}{c}{${ }^{2}$} \\
& N & $\%$ & N & $\%$ & N & $\%$ \\
\hline Sim & 31 & 41,3 & 44 & 58,7 & 75 & 100,0 \\
Feminino & 21 & 46,7 & 24 & 53,3 & 45 & 100,0 \\
Masculino & 10 & 33,3 & 20 & 66,7 & 30 & 100,0 \\
Não & 236 & 48,2 & 254 & 51,8 & 490 & 100,0 \\
Feminino & 166 & 54,2 & 140 & 45,8 & 306 & 100,0 \\
Masculino & 70 & 38,0 & 114 & 62,0 & 184 & 100,0 \\
\hline
\end{tabular}

Tabela 5. Distribuição dos alunos estudados segundo categorias de excesso ponderal, sexo e classificação de pressão arterial.

\begin{tabular}{cccccccc}
\hline & \multicolumn{4}{c}{ Pressão arterial } & \multicolumn{2}{c}{ Total } \\
Excesso ponderal & \multicolumn{2}{c}{ Alterada } & \multicolumn{2}{c}{ Não alterada } & \multicolumn{2}{c}{${ }^{2}$} & Nota \\
& N & $\%$ & N & $\%$ & N & $\%$ \\
\hline Sim & 31 & 41,3 & 44 & 58,7 & 75 & 100,0 \\
Feminino & 21 & 46,7 & 24 & 53,3 & 45 & 100,0 \\
Masculino & 10 & 33,3 & 20 & 66,7 & 30 & 100,0 \\
& & & & & & \\
Não & 236 & 48,2 & 254 & 51,8 & 490 & 100,0 \\
Feminino & 166 & 54,2 & 140 & 45,8 & 306 & 100,0 \\
Masculino & 70 & 38,0 & 114 & 62,0 & 184 & 100,0 \\
\hline
\end{tabular}

\section{DISCUSSÃO}

A prevalência de níveis pressóricos alterados $(45,3 \%)$ no grupo avaliado merece destaque. Quando analisado por sexo, maior preocupação 
merecem as meninas (53,3\%). A I Diretriz de Prevenção da Aterosclerose na Infância e Adolescência $^{(10)}$ aponta uma prevalência de 8,2\% para hipertensão arterial, dados que se aproximam dos 7,7\% encontrados em 1253 escolares de Maceió(19). Por outro lado, esses achados citados são muito inferiores aos encontrados no presente estudo, o qual se aproxima da situação detectada em outro estudo realizado no Ceará, na cidade de Fortaleza(20), com 342 crianças e adolescentes de 6 a 18 anos de ambos os sexos, que detectou uma prevalência de $44 \%$ para hipertensão arterial. Valores tão discrepantes muitas vezes levantam questionamentos sobre falhas metodológicas ou relacionadas à qualidade dos equipamentos utilizados. No entanto os procedimentos aqui adotados seguiram protocolos normatizados pelas $\mathrm{V}$ Diretrizes Brasileiras de Hipertensão Arterial|(12) , assim como o equipamento utilizado também foi adequado. $O$ fato de haver outro estudo no Ceará com valores semelhantes aos aqui encontrados leva a crer que a situação local realmente merece preocupação maior do que a verificada em outros locais.

$\mathrm{A} \mathrm{OMS}^{(5)}$ chama a atenção para as prevalências de sobrepeso entre crianças e adolescentes, que dobrou de 1973 a 1983 e quadriplicou de 1983 a 1994. A mesma tendência é citada para o Japão, com crianças em idade escolar e prevalência aumentada de $5 \%$ para $10 \%$ em sobrepeso e de $1 \%$ para $2 \%$ em obesidade.

Outros valores também preocupantes são apontados em pesquisa realizada em 2002$2003^{(11)}$, onde se constatou, nas regiões NorteNordeste, sobrepeso em $11,8 \%$ do sexo masculino e em $11,6 \%$ do sexo feminino e obesidade em $1,1 \%$, de ambos os sexos.

O presente estudo aponta uma prevalência de $9,6 \%$ para sobrepeso e $3,7 \%$ para obesidade em ambos os sexos, indo de encontro a outros estudos na mesma região, como em Fortaleza com prevalências encontradas entre escolares da rede pública de ensino de $8,30 \%$ para sobrepeso e $3,35 \%$ para obesidade ${ }^{(21)}$. Na periferia da cidade de Fortaleza foi encontrada uma prevalência de excesso ponderal mais baixa que a aqui apontada, com $5,5 \%$ para as meninas e $8,2 \%$ para os meninos, chamando a atenção para fatores de influencia, como a situação sócio-econômica e o contexto social ${ }^{(22)}$. Quando investigadas escolas da rede particular de ensino, ainda em Fortaleza, o excesso ponderal aumentou consideravelmente e demonstrou uma realidade bem mais alarmante, com prevalências de $19,5 \%$ a 38\%23-24.

Os dados discrepantes dentro da mesma cidade e diferindo de acordo com a abrangência dos estudos e com a época de realização dos mesmos, mostram que a problemática do excesso ponderal na infância e adolescência deve ser tratada de forma particularizada, caso se deseje que os resultados das pesquisas viabilizem uma tomada de ações de intervenção para reverter sua prevalência ou evitar seu agravamento. No caso do presente estudo, os dados gerados merecem atenção, pois não há mapeamento nutricional da região de Caucaia, publiciado em periódicos indexados, que sirva de base para tomada de decisão em políticas públicas necessárias.

Avaliando o outro extremo de comprometimento nutricional, a desnutrição, os valores também chamam a atenção, embora os presentes achados, de $7,4 \%$ entre os alunos do sexo feminino e $9,4 \%$ entre os do sexo masculino, fiquem abaixo dos valores encontrados em estudo realizado entre adolescentes de Fortaleza, onde as prevalências foram de $9,3 \%$ e $15,5 \%$, respectivamente, entre meninas e meninos ${ }^{(22)}$.

A prevalência de desnutrição apontada na Pesquisa de Orçamentos Familiares - POF 20022003 , realizada pelo $\operatorname{IBGE}^{(11)}$, foi de $7,4 \%$ entre adolescentes nordestinos, mas a mesma não foi estratificada por cidade e estado, limitando assim uma análise mais individualizada.

De um perfil anterior, caracterizado pela desnutrição, o Brasil está passando para um perfil de crianças e adolescentes obesos ou com sobrepeso, assim como outros países em desenvolvimento $^{(25)}$. O Nordeste também vê essa realidade acontecer nos poucos dados que oferece.

No grupo avaliado a questão da prática de atividade física não parece preocupante, pois a maioria foi considerada ativa ou muito ativa. Os níveis pressóricos dos adolescentes avaliados não foram associados à atividade física e ao estado nutricional contrariando vários estudos publi- 
cados, que associam níveis pressóricos alterados com o sedentarismo, excesso ponderal, e padrão alimentar inadequado(26-27-28-29-30-31-32-33-34-35).

Vale ressaltar que para que a prática da atividade física tenha influência no excesso ponderal e nos índices pressóricos é necessário que a intensidade seja adequada e suficiente, como demonstrou estudo realizado em Pernambuco com adultos que foram atletas na adolescência e atualmente praticam apenas atividade física de lazer, onde não se encontrou associação entre as variáveis estudadas ${ }^{(36)}$.

Sabe-se também que determinantes da alteração pressórica não são exclusivamente ambientais, como os hábitos e estilo de vida, havendo também influências genéticas, de raça, idade e sexo ${ }^{(23)}$. Não foi objetivo do presente estudo investigar tais aspectos, pois o que se pretendia era conhecer uma realidade para viabilizar ações de intervenção, caso necessário.

Nessa perspectiva, a prática de atividade física pode ser estimulada, mas no sentido de reforçar um comportamento já adequado para a maioria dos adolescentes avaliados.

Uma limitação do estudo não poderia deixar de ser apontada ao finalizar a presente discussão sendo representada tanto pelo número de perdas da amostra pretendida, como pelo fato de se tratar de uma pesquisa realizada em uma única escola, impedindo generalizações. Embora não possa ser avaliado, pode ser que o contingente de alunos que se recusaram a participar fosse constituido por aqueles mais obesos e/ou inativos. De qualquer forma, é importante assinalar que o número de estudantes aqui avaliados foi alto, sendo provável que a situação se reproduza em outras pesquisas.

Fica claro então que existe a necessidade de mais estudos em regiões metropolitanas e longe das capitais para que se possa suprir a necessidade de um banco de dados que desvele a realidade nutricional e dos fatores de risco cardiovasculares envolvidos em populações específicas de crianças e adolescentes. Prevalências elevadas demandam obrigatoriamente a investigação da presença de fatores de risco cardiovascular, principalmente os associados a estilo de vida, como atividade física e alimentação.

\section{CONCLUSÃO}

A prevalência de níveis pressóricos alterados (pré-hipertensão e hipertensão arterial) foi alta $(45,3 \%)$ no grupo estudado, principalmente no sexo feminino. A prevalência de excesso ponderal encontrada $(13,5 \%)$ corrobora dados nacionais e locais. Os adolescentes exibiram perfil adequado de atividade física, ativos ou muito ativos (70,3\%).

Não houve, no momento, associação entre hipertensão arterial, prática de atividade física e situação ponderal, o que não minimiza o fato de que a situação encontrada entre os adolescentes estudados configura risco à saúde.

Nesse contexto, os adolescentes avaliados necessitam de urgente encaminhamento a serviços de saúde, a fim de investigar de forma mais aprofundada seus elevados níveis pressóricos e respectivos determinantes destes, com a implementação de ações de controle. Tal conduta contribuirá para a prevenção de complicações na vida adulta.

\section{REFERÊNCIA BIBLIOGRÁFICAS}

1. Bracco $M M$, Carvalho $K M B$, Bottoni $A$, Nimer $M$, Gagliannone CP, Taddei JAAC, et al. Atividade Física na Infância e adolescência: Impacto na Saúde pública. Revista Ciências Médicas. 2003; 12(1): 89-97.

2. Araujo TL, Lopes MVO, Cavalcante TF, Guedes NG, Moreira RP, Chaves ES, et al. Análise de indicadores de risco para hipertensão arterial em crianças e adolescentes. Revista Escola de Enfermagem USP. 2008; 42(1): 120-6.

3. Costa MC, Silva MCM, Santos JS, Teles C, Souza KEP, Melo BO. Estilo de vida de adolescentes: consumo alimentar, de bebida alcoólica e atividade física em Teixeira de Freitas - BA. Revista Baiana de Saúde Pública. 2004; 28(2): 151-166.

4. Gambardela AMD, Frutoso MFP, Franch C. Prática Alimentar de Adolescentes. Revista de Nutrição. 1999; 12(1): 55-63.

5. Organização Mundial da Saúde. Prevenindoe Controlando a Epidemia Global. São Paulo: Roca; 2004. 
6. Rosa MLG, Mesquita ET, Rocha ERR, Fonseca VM. Índice de massa Corporal e circunferência da cintura como marcadores de Hipertensão Arterial em Adolescentes. Arquivos Brasileiros de Cardiologia. 2007; 88(5): 573-8.

7. Silva MA, Rivera IRR, Ferraz MRMT, Pinheiro AJT, Alves SWS, Moura AA, et al. Prevalência de Fatores de Risco Cardiovascular em Crianças e Adolescentes da Rede de Ensino da Cidade de Maceió. Arquivos Brasileiros de Cardiologia. 2005;84(5): 387-92.

8. Souza GS, Duarte MFS. Estágios de mudança de comportamento relacionados à atividade física em adolescentes. Revista Brasileira de Medicina do Esporte. 2005; 11(2): 104-8.

9. HEYWARD, V. H. Avaliação Física e Prescrição de Exercício Técnicas avançadas. Porto Alegre: Artmed; 2004.

10. Sociedade Brasileira de Cardiologia. I Diretriz de prevenção da Aterosclerose na Infância e Adolescência. Arquivos Brasileiros de Cardiologia. 2005; 85(6): 3-36.

11. Instituto Brasileiro de Geografia e Estatística (homepage na internet). Pesquisa de Orçamentos Familiares - POF 2002/2003 (citado 2008 julho 10). Disponivel em http://www.ibge.gov.br

12. Sociedade Brasileira de Cardiologia, Sociedade Brasileira de Hipertensão, Sociedade Brasileira de Nefrologia. V Diretrizes Brasileiras de Hipertensão Arterial.Revista da Sociedade Brasileira de Hipertensão. 2006; 9(4): 1-49.

13. US Department of Health and Human Services, National Institutes of Health, National Heart, Lung and Blood Institute. The Fourth Report on the Diagnosis, Evaluation, and Treatment of High Blood Pressure in Children and Adolescents. 2005 ; 5: 5267.

14. US Department of Health and Human Services, National Institutes of Health, National Heart, Lung and Blood Institute. Your guide to lowering your blood pressure with DASH; DASH eating plan lower your blood pressure. 2006; 6: 4082.

15. Center for Disease Control and Prevention and Health Promotion (homepage na internet) (citado 2008 junho 18). Disponível em www.cdc.gov/growthchart.
16. IPAQ Guidelines for data processing and analysis of the international physical activity questionnaire:Short and Long Forms (homepage na internet) (citado 2009 janeiro 07). Disponivel em http://www.ipaq.ki.se

17. Guedes DP, Lopes CC, Guedes JERP. Reprodutibilidade e validade do Questionário Internacional de Atividade Física em adolescentes. Revista Brasileira Medicina Esporte. 2005; 11(2): 151-8.

18. PASW - Predictive Analytics Software for Windows v. 17.0.

19. Silva MAM, Rivera IR, Ferraz MRMT, Pinheiro AJT, Alves SWS, Moura AA. Prevalência de Fatores de Risco Cardiovascular em Crianças e Adolescentes da Rede de Ensino da Cidade de Maceió. Arquivos Brasileiros de Cardiologia. 2005; 84(5): 387-92

20. Silva ARV, Damasceno MMC, Marinho NBP, Almeida LS, Araújo MFM, Almeida PC, et al. Hábitos alimentares de adolescentes de escolas públicas de Fortaleza, CE, Brasil. Revista Brasileira de Enfermagem. 2009; 62(1): 18-24.

21. Araújo TL, Lopes MVO, Cavalcante TF, Guedes NG, Moreira RP, Chaves ES, et al. Análise de indicadores de risco para hipertensão arterial em crianças e adolescentes. Revista Escola de Enfermagem USP. 2008; 42(1): 120-6.

22. Sampaio HAC, Sabry MOD, Azevedo DV, Diniz DB, Passamai MPB, Sá MLB, et al. Educação Nutricional na prevenção e controle de doenças: um modelo para aplicação no programa saúde da família e no currículo de ensino fundamental e médio. Relatório Final;2007.

23. Pereira RM. Prevalência de obesidade em crianças e adolescentes de 7 a 14 anos em escolas públicas, Fortaleza-CE (dissertação de mestrado). Fortaleza: Universidade Estadual do Ceará; 2005.

24. Campos LA, Leite AJM, Almeida PC. Prevalência de sobrepeso e obesidade em adolescentes escolares do município de Fortaleza, Brasil. Revista Brasileira de Saúde Materno Infantil. 2007; 7(2): 183-190.

25. Santana LMDM. Ocorrência de Síndrome Metabólica entre escolares e fatores dietéticos associados (dissertação de mestrado) Fortaleza: Universidade Estadual do Ceará; 2006. 
26. Monteiro CA, Mondini L, Souza AL, Popkin BM, Popkin BM. European Journal of Clinical Nutrition. 1995; 49(2): 105-13.

27. Kuschnir MCC, Mendonça GAS. Fatores de risco associados à hipertensão arterial em adolescentes. Jornal de Pediatria. 2007; 83(4): 335-42.

28. Alves JGB, Siqueira PP. Excesso de peso e inatividade física em crianças moradoras de favelas na região metropolitana do Recife, $\mathrm{PE}$. Jornal de Pediatria. 2009: 85(1): 67-71.

29. Rodrigues NA, Perez AJ, Pirez JGP, Carletti L, Araújo MTM, Moyses MR,et al. Fatores de risco cardiovasculares, suas associações e presença de síndrome metabólica em adolescentes. Jornal de Pediatria. 2009; 85(1): 55-60.

30. FernandezAC, Mello MT, Tufik S, Castro PM, Fisberg $M$. Influência do treinamento aeróbio e anaeróbio na massa de gordura corporal de adolescentes obesos. Revista Brasileira de Medicina do Esporte. 2004; 10(3): 152-8.

31. Mascarenhas LPG, Salgueirosa FM, Nunes GF. Relação entre diferentes índices de atividade física e preditores de adiposidade em adolescentes de ambos os sexos. Revista Brasileira de Medicina do Esporte. 2005; 11(1): 214-8.

32. Pereira A, Guedes AD, Verreschi ITN, Santos RD, Martinez TLRA. A obesidade e sua associação com os demais fatores de risco cardiovascular em escolares de Itapetininga, Brasil. Arquivos Brasileiros em Cardiologia. 2009; 93(3): 253-60.
33. Longo GZ, Neves J, Luciano VM, Perez MA. Prevalência de níveis pressóricos elevados e fatores associados em adultos de Lages/SC. Arquivos Brasileiros de Cardiologia. 2009; 93(4) 387-94.

34. Costa GB, Horta N, Resende ZF, Souza G, Barreto LMF, Correia LH, et al. Índice de Massa Corporal apresenta boa correlação com o perfil próaterosclerótico em crianças e adolescentes. Arquivos Brasileiros de Cardiologia. 2009; 93(3): 261-7.

35. Becker MMC, Silva OB, Moreira IEG, Vitor EG. Pressão Arterial em adolescentes durante teste ergométrico. Arquivos Brasileiros de Cardiologia. 2007; 88(3): 329-33.

36. Sabia RV, Santos JE, Ribeiro RPP. Efeito da atividade física associada à orientação alimentar em adolescentes obesos: comparação entre o exercicio aeróbio e anaeróbio. Revista Brasileira de Medicina do Esporte. 2004; 10(5): 349-355.

37. Alves JGB, Montenegro, FMU, Oliveira FA. Prática de esportes durante a adolescência e atividade física de lazer na vida adulta. Revista Brasileira Medicina Esporte. 2005; 11(5): 291-4.

Endereço para correspondência:

Claudia dos Reis Lisboa Lisboa, Educadora Física, Mestre em Saúde Pública pela Universidade Estadual do Ceará, CE. - Currículo plataforma lattes CNPq - End. Rua Seu Né Bezerra, 1620 Casa 110 - Bairro Costa e Silva - Mossoró - RN - telefone (84) 99048007 e (85) 86330102 kallsport@yahoo.com.br 